\title{
Phase Structure and Cyclic Deformation in Eutectic Tin-Lead Alloy: A Numerical Analysis
}

\author{
Y.-L. Shen ${ }^{1 *}$, W. $\mathrm{Li}^{1}$ and H. E. Fang ${ }^{2}$ \\ ${ }^{1}$ Department of Mechanical Engineering \\ The University of New Mexico \\ Albuquerque, NM 87131 \\ ${ }^{2}$ Sandia National Laboratories \\ Albuquerque, NM 87185
}

\begin{abstract}
\end{abstract}

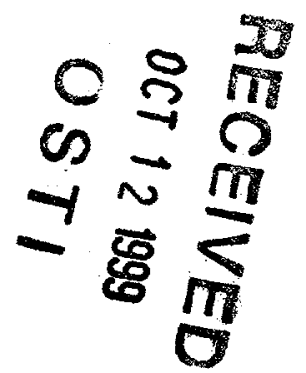

This study is devoted to providing a mechanistic rationale of coarsening induced failure in solder alloys during thermomechanical fatigue. Micromechanical modeling of cyclic deformation of eutectic tin-lead alloy was undertaken using the finite element method. The models consist of regularly arranged tin-rich and lead-rich phases, simulating the lamellar array and colony structure in a typical eutectic system. A fine structure and a coarse structure, bearing the same phase fraction but different in the aspect ratio of each lead-rich layer and in the number of lead-rich layers in each colony, are utilized for representing the microstructure before and after coarsening, respectively. Both phases are treated as elastic-plastic solids with their respective properties. For simplicity the creep effect is ignored without compromising the main objective of this study. Cyclic loading under pure shear and uniaxial conditions is modeled. It is found that both the fine and coarse structures exhibit essentially the same macroscopic stress-strain response. The coarse structure, however, shows a greater maximum effective plastic strain on a local scale throughout the deformation. The numerical result implies that, in a solder joint, a locally coarsened region may not be mechanically weaker than its surrounding, but it is subject to early damage initiation due to accumulated plasticity. Other implications regarding solder alloy failure and micromechanical modeling of two-phase materials are discussed.

\footnotetext{
${ }^{*}$ Corresponding author
} 


\section{Introduction}

Tin $(\mathrm{Sn})$-lead $(\mathrm{Pb})$ alloys with near eutectic compositions have been widely used as soldering materials in the package of semiconductor and communication devices. Although they possess good manufacturability essential for soldering (low melting point, easy flow, good wetting capability on typical metallizations such as copper, silver and gold, and acceptable chemical characteristics), their long-term structural reliability has always been a major concern. One of the main reliability issues is thermomechanical fatigue. Stresses in solders are generated due to the thermal expansion difference of the components they connect, as a consequence of temperature fluctuations stemming from the operation environment. Material degradation is caused not only by cyclic straining, but also by creep damage because of the inherent low melting point such that even room temperature is sufficient to trigger high temperature deformation mechanisms. These are further compounded by the fact that the alloy microstructure is changing with time, even at room temperature, with or without applied stresses. A common type of damage is the formation of a microstructurally coarsened band (with greater sizes of phase domains and grains) along which a crack forms, leading to ultimate failure [1-5]. Although this problem has been existent for a long time, the evolution of damage and its underlying mechanisms are still not well understood.

From a metallurgical viewpoint, a coarser microstructure is typically thought to be weaker in response to mechanical loading, i.e., the Hall-Petch type behavior. If this can be applied to thermomechanical fatigue of $\mathrm{Sn}-\mathrm{Pb}$ solder, it would be natural to argue that once a coarsened band has formed, further deformation should become concentrated along the band due to its low strength with eventual failure along the band. However, this simple view may not accurately reflect what occurs, because the relation between strength and phase size of eutectic $\mathrm{Sn}-\mathrm{Pb}$ is still subject to great uncertainty. Under almost all application conditions the homologous temperature for this alloy is well above 0.5 , so the validity of the Hall-Petch type relation is questionable. For instance, diffusional creep results in an increasing creep rate with decreasing grain size [6], and grain/phase boundary sliding (which has been observed in eutectic $\mathrm{Sn}-\mathrm{Pb}$ ) is a direct manifestation that the boundary region is weaker so the strength increases with increasing grain/phase size. Direct experimental characterizations of strength variation with coarsening have shown conflicting results. We have conducted microhardness testing on newly solidified $\mathrm{Sn}-\mathrm{Pb}$ alloy and found that the 


\section{DISCLAIMER}

This report was prepared as an account of work sponsored by an agency of the United States Government. Neither the United States Government nor any agency thereof, nor any of their employees, make any warranty, express or implied, or assumes any legal liability or responsibility for the accuracy, completeness, or usefulness of any information, apparatus, product, or process disclosed, or represents that its use would not infringe privately owned rights. Reference herein to any specific commercial product, process, or service by trade name, trademark, manufacturer, or otherwise does not necessarily constitute or imply its endorsement, recommendation, or favoring by the United States Government or any agency thereof. The views and opinions of authors expressed herein do not necessarily state or reflect those of the United States Government or any agency thereof. 


\section{DISCLAIMER}

Portions of this document may be illegible in electronic image products. Images are produced from the best available original document. 
hardness value increases with microstructural coarsening over a period of three months at room temperature after solidification [7]. An initially coarser structure also showed greater hardness values than the finer structure. These results suggest that an "inverse Hall-Petch" behavior exists. Our finding is consistent with another study on a rolled $\mathrm{Sn}-\mathrm{Pb}$ alloy with uncontrolled initial microstructure [8], where an increase of microhardness with a coarsening phase structure after annealing at $125^{\circ} \mathrm{C}$ was observed. In addition, in a creep test conducted at room temperature of the same material [9], a decrease in phase size resulted in an increased creep rate for the same applied stress. All of these observations suggest that a coarser microstructure has higher mechanical strength. On the other hand, experiments showing the trend conforming to the Hall-Petch type behavior have been reported. Chill-cast, near-eutectic $\mathrm{Sn}-\mathrm{Pb}$ alloys have shown an overall decrease in strength and hardness with time [10,11], with some scatter during the first few days [10].

In the present study, no attempt is made to resolve the above issue. Our objective is to computationally examine how the phase morphology itself affects the overall mechanical response and damage processes. The micromechanisms of deformation at the length scale of grains and grain boundaries are intentionally ignored. By devising idealized "fine" and "coarse" structures in the similitude of lamellar arrangements typical of eutectic alloys, we seek to model, within the continuum framework, the effects of phase coarseness on the effective mechanical response and the evolution of local field quantities. A mechanistic rationale can then be provided for the experimental fact that fatigue damage tends to develop within the coarsened region. We believe that the present approach, albeit simple, is very useful in gaining insight into the failure initiation in solder joints, which is a very complex problem by nature.

\section{Approach}

The typical microstructure of an eutectic $\mathrm{Sn}-\mathrm{Pb}$ alloy consists of alternating layers of a $\mathrm{Pb}$-rich $\alpha$ phase solid solution and a Sn-rich $\beta$-phase solid solution, with the $\mathrm{Pb}$-rich phase appearing to be embedded within the $\mathrm{Sn}$-rich matrix. Near room temperature the solid solubility of $\mathrm{Pb}$ in $\mathrm{Sn}$ is close to zero and of $\mathrm{Sn}$ in $\mathrm{Pb}$ is less than $3 \mathrm{wt} \%$. Layers oriented in essentially the same direction form a "colony". The Sn-rich matrix consists of grains smaller than the overall feature size of the dual phase structure. In the present modeling, the grain structure is neglected, and a periodic and regular 
arrangement of the colony structure is assumed. Figures 1(a) and 1(b) show the model geometry for the "fine" and "coarse" structures, respectively. The two-dimensional computational domain is actually a quadrant of the representative volume element (unit cell), and is taken to be a square of side length $l_{0}$. A mirror symmetry exists across the four boundaries. Therefore the model consists of four regions each representing a quarter of a colony. In the fine and coarse structures, the aspect ratios of each $\mathrm{Pb}$-rich domain are arbitrarily taken to be 9 and 3.75, respectively. A total of 5 and 3 layers of $\mathrm{Pb}$-rich phase exist in each colony for the fine and coarse structures, respectively. In the actual material, the volume fraction of the $\mathrm{Pb}$-rich phase is approximately 0.3 . In the present twodimensional model we assume an area fraction of $\mathrm{Pb}$-rich phase to be 0.45 in both the fine and coarse structures. The plane strain condition (with no displacement in the $z$ direction) is used in all calculations. It is worth stressing that our concern is not to simulate any structure containing a coarsened region surrounded by a finely structured matrix. Attention here is devoted to the independent mechanical characterization of a "coarse" structure and a "fine" structure, each with a spatially extended two-phase layout.

In all calculations the following boundary conditions are used.

$$
u_{y}=0 \text { and } \tau_{x}=0 \quad \text { on } y=0
$$

and

$$
u_{x}=0 \quad \text { and } \tau_{y}=0 \quad \text { on } \quad x=0,
$$

where $u_{x}$ and $u_{y}$ represent the displacement components along the $x$ and $y$ directions, respectively, and $\tau_{x}$ and $\tau_{y}$ represent the traction components. Two types of loading are considered, namely pure shear and uniaxial loading. In the first case, the prescribed normal displacements $-\Delta l$ and $\Delta l$ are imposed on the right and top boundaries, respectively, so during deformation

$$
\begin{array}{llll}
u_{x}=-\Delta l & \tau_{y}=0 & \text { on } & x=l_{0}, \\
u_{y}=\Delta l & \tau_{x}=0 & \text { on } & y=l_{0} .
\end{array}
$$

This gives essentially the pure shear condition. In uniaxial loading a prescribed displacement is imposed on the top boundary, so

$$
\begin{array}{rrrr} 
& u_{y}=\Delta l \quad & \text { on } \quad y=l_{0}, & \\
\tau_{y}=0 \quad \text { and } \int \tau_{x} \mathrm{~d} y=0 & \text { with } u_{x}=d \quad \text { on } x=l_{0},
\end{array}
$$


where $d$ is determined by the analysis. Note that we refer to this loading mode as "uniaxial" only in a two-dimensional sense, since the plane strain condition is enforced.

No thermal effects are considered in this study. Both phases are characterized as isotropic elastic-plastic solids, with rate-independent Mises plasticity and no hardening. Although a temperature- and time-dependent plastic behavior is expected for both the actual $\mathrm{Sn}$-rich and $\mathrm{Pb}$-rich solid solutions, our objective here is to extract the baseline information by undertaking a straightforward approach such that the constitutive model has the fewest material parameters to adjust. At room temperature under normal quasi-static loading conditions, both $\mathrm{Sn}$ and $\mathrm{Pb}$, as well as the $\mathrm{Sn}-\mathrm{Pb}$ alloy display a near elastic-perfectly plastic behavior. The material properties of the Sn-rich and $\mathrm{Pb}$-rich phases, extracted from reference [12], used in the modeling are: $E_{\mathrm{Sn}}=50 \mathrm{GPa}$, $v_{\mathrm{Sn}}=0.33, \sigma_{\mathrm{o}, \mathrm{Sn}}=11 \mathrm{MPa}, E_{\mathrm{Pb}}=28.1 \mathrm{GPa}, v_{\mathrm{Pb}}=0.39$ and $\sigma_{\mathrm{o}, \mathrm{Pb}}=10 \mathrm{MPa}$, where $E, v$ and $\sigma_{\mathrm{o}}$ stand for Young's modulus, Poisson's ratio and yield strength, respectively.

The general-purpose finite element program ABAQUS [13] was employed in the calculation. The discretizations used 4-noded quadrilateral elements. The convergence of results was checked through various degrees of mesh refinement. All the results presented in this paper are based on calculations with 6400 elements. It was found that further mesh refinement resulted in insignificant changes in the overall stress-strain response and the local field quantities.

For presentation of the results, some stress and strain quantities need to be defined. In this paper we refer to the applied overall strain and the overall stress as macroscopic strain and macroscopic stress, respectively. The macroscopic strains, $E_{x x}$ and $E_{y y}$, are defined to be the normal displacements at the right and top boundaries, respectively, divided by $l_{0}$. The macroscopic stresses are

$$
\Sigma_{x x}=F_{x} / l_{0}
$$

and

$$
\Sigma_{y y}=F_{y} / l_{0},
$$

where $F_{x}$ and $F_{y}$ are the sums of normal nodal forces induced on the right and top boundaries, respectively, with a unit of force per length. 


\section{Results}

\subsection{Pure Shear Loading}

In this section we present the numerical results of cyclic pure shear loading. The macroscopic strain history in each cycle for $E_{x x}$ is $0 \rightarrow-0.0025 \rightarrow 0.0025 \rightarrow 0$ and for $E_{y y}$ is $0 \rightarrow 0.0025 \rightarrow-0.0025 \rightarrow$ 0 , each imposed independently but synchronously. Therefore it is essentially a pure-shear loading which is considered most important in solder joints. Figure 2 shows the curves of macroscopic stress $\left(\Sigma_{y y}\right)$ vs. macroscopic strain $\left(E_{y y}\right)$, for both the fine and coarse structures. In this case it is also the shear stress-shear strain response. Only the first loading cycle is shown, since further cycling simply results in repeated hysteresis loops. It is clear that the fine and coarse structures follow essentially the same response. Therefore, one can infer that if a locally coarsened microstructure has developed within the initially fine structure, the coarsened region is neither a weaker nor a stronger part of the material. Of course, this conclusion is predicated upon the validity of our continuum approach, along with the underlying assumptions.

We now turn to the following question: is our model still able to rationalize the fact that mechanical damage preferentially occurs within the coarsened region? Figures 3(a) and 3(b) show the contours of constant effective plastic strain within the $\mathrm{Sn}$-rich phase and $\mathrm{Pb}$-rich phase, respectively, for the fine structure at the end of the first loading cycle. The corresponding plots for the coarse structure are shown in Figures 4(a) and 4(b). In all cases, the entire material has undergone plastic deformation, as revealed from the macroscopic stress-strain curves shown in Fig. 2. The local deformation pattern, however, is highly non-uniform. In the fine structure (Fig. 3), the plastic flow path is severely disturbed so deformation tends to localize into many small regions. In the coarse structure (Fig. 4), the less disturbed flow path leads to a banded pattern. In both the fine and coarse structures, the maximum effective plastic strain in the Sn-rich phase is moderately greater than that in the $\mathrm{Pb}$-rich phase. We also found that, immediately from the onset of plastic yielding, the maximum plastic strain appears at the same location throughout the cyclic deformation history. Therefore we can make a direct comparison of the maximum plastic strain during deformation for the two structures. 
Figure 5 shows the variations of maximum effective plastic strain with the applied macroscopic strain $E_{y y}$, for the fine and coarse structures during the first full cycle. The very short horizontal segment at the beginning of each loading/unloading phase is due to the elastic portion of the loading. It is evident that the coarse structure shows a higher level of effective plastic strain than the fine structure at all stages. The difference increases as the deformation progresses. This trend continues with further cycling, which is not shown in the figure. Note that although the fine and coarse structures show essentially the same macroscopic response, the local evolution of plastic strain in the coarse structure is increasingly stronger than that in the fine structure. The effective plastic strain, representing the accumulation of irrecoverable deformation in an elastic-plastic solid, is a parameter directly related to the propensity of damage initiation (e.g., formation of microvoids or microcracks in ductile solids). It can thus be inferred that if a coarse structure has formed and is subject to the same macroscopic loading condition as in the surrounding fine structure, the coarse structure is more prone to fatigue damage. This is in qualitative agreement with experimental observations.

\subsection{Uniaxial Loading}

In an attempt to examine the generality of the numerical result in Section 3.1, we have also carried out an analysis of uniaxial loading. Here the displacement along the $y$ direction is imposed on the top boundary. The macroscopic strain history in each cycle is $E_{y y}=0 \rightarrow 0.0025 \rightarrow-0.0025 \rightarrow 0$. Figure 6 shows the macroscopic stress $\left(\Sigma_{y y}\right)$-strain $\left(E_{y y}\right)$ response for the fine and coarse structures during the first cycle. As in the case of pure shear loading, the fine and coarse structures show essentially the same mechanical response. In addition, the evolution of local effective plastic strain (not shown here) displays essentially the same pattern as in the pure shear loading (Figs. 3 and 4), except the strain values are slightly smaller in this uniaxial loading case. The maximum plastic strain always occurs at the same location in the Sn-rich phase throughout the cycling history. Figure 7 shows the variations of maximum effective plastic strain with the applied macroscopic strain. As in Section 3.1, the coarse structure shows significantly stronger plasticity than the fine structure. The difference increases further with further cycling. It thus appears that the uniaxial loading exhibits the same micromechanical features as in the case of pure shear. 


\section{Discussion}

Micromechanical modeling of idealized eutectic $\mathrm{Sn}-\mathrm{Pb}$ microstructures bearing the "fine" and "coarse" phase arrangements were carried out. We have found that a coarser microstructure is neither stronger nor weaker than a finer microstructure in terms of the overall stress-strain response. For the sake of argument, the present model seems to render a "neutral" position with reference to the apparent controversy of experimental results cited in Section 1. Attention is then turned to the numerical finding that the maximum effective plastic strain is always larger in the coarser structure. Consequently, once a locally coarsened band becomes existent, the faster accumulation of plasticity there will cause early failure, compared to the rest of the material with still a finer microstructure. Fatigue failure of the entire solder joint thus appears along the coarsened band. It should be noted that in this study we do not consider how the coarsened band is formed. It may involve the interaction of mechanical and metallurgical factors such as strain enhanced diffusion and dynamic recovery/recrystallization $[4,7]$. Further studies are needed to clarify this.

It is worth mentioning that, in addition to the stronger plasticity observed in the coarser structure, the numerical result also suggests that the colony boundary is more prone to damage initiation. This can be seen from Figs. 3(a) and 4(a), where the maximum plastic strain occurs at colony boundaries in both structures.

Since the present study involves modeling the two-phase microstructure within the continuum framework, it actually bears some resemblance to the micromechanical models frequently adopted for simulating metal matrix composites, with the current $\mathrm{Pb}$-rich phase viewed as the reinforcing inclusions. The major difference is that the inclusion phase in metal matrix composites is normally a purely elastic material with high stiffness. In the present case the $\mathrm{Pb}$-rich phase is a ductile one, as well as the matrix Sn-rich phase. Furthermore, in most composites modeling the inclusion layout is not as complex as in the present work. It has been shown that, in metal matrix composites, the macroscopic stress-strain response is strongly influenced by the shape and spatial distribution of the inclusion phase for the same inclusion concentration [14,15]. Curiously, there is virtually no difference in the present $\mathrm{Sn}-\mathrm{Pb}$ case. We have explored this effect by taking the $\mathrm{Pb}$-rich "inclusion" to be purely elastic or elastic-plastic with a much higher yield strength of $40 \mathrm{MPa}$, with all the other 
material parameters remaining the same. Figure 8 shows the macroscopic stress-strain curves resulting from such modeling, for both the fine and coarse structures under uniaxial tension loading. It is clear that with an elastic inclusion phase, the difference in plastic flow stress between the fine and coarse structures becomes very large. For an inclusion phase of yield strength $40 \mathrm{MPa}$, the difference becomes smaller. Thus, for the modeling of $\mathrm{Sn}-\mathrm{Pb}$ alloy presented in Section 3 , the same macroscopic response obtained for the fine and coarse structures is due to the very soft nature of the $\mathrm{Pb}$-rich phase. In fact, in this work the yield strength of the Pb-rich phase (10 $\mathrm{MPa})$ is almost identical to that of the Sn-rich matrix (11 MPa). Nevertheless, such a small mismatch, along with the somewhat larger elastic mismatch between $\mathrm{Sn}$ and $\mathrm{Pb}$ (see Section 2) still results in a significant difference in the evolution of local effective plastic strain presented in Section 3.

As a side note, the two curves in Fig. 8 labeled "elastic inclusion" can be used to illustrate a metallurgical feature in another important alloy system. In carbon steels, i.e. the iron (Fe)-carbon (C) system, the micro-constituent "pearlite" is an eutectoid structure composed of alternating layers of cementite $\left(\mathrm{Fe}_{3} \mathrm{C}\right.$ ) and ferrite matrix (Fe-rich solid solution) pertaining to a colony-type arrangement. The phase morphology is basically the same as the eutectic structure. In our model, if one thinks of the inclusion as cementite (elastic) and the matrix as ferrite (elastic-plastic), then Fig. 8 illustrates, from a micromechanical viewpoint, that a finer structure is stronger than a coarser structure. Indeed, by controlling the heat treatment, metallurgists can produce "fine pearlite" which shows a higher strength than "coarse pearlite".

\section{Conclusions}

By employing a micromechanical model simulating the microstructural phase morphology in eutectic composition, we have numerically modeled the mechanical characteristics of $\mathrm{Sn}-\mathrm{Pb}$ alloy during cyclic deformation. The finer and coarser phase structures show essentially the same macroscopic stress-strain response during both the pure shear and uniaxial loading. The implication is that local phase coarsening does not necessarily induce a locally weaker region in actual solder joints, on the basis of our continuum approach. The evolution of local field quantities, however, reveals that the maximum effective plastic strain is always larger in the coarser structure throughout the cycling history. Damage is thus more prone to initiate in the coarse structure, which can lead to 
eventual fatigue failure along the coarsened band in solder joints. The strongest plasticity effect is seen to appear in the Sn-rich phase at the colony boundaries.

\section{Acknowledgment}

Valuable discussions with Professors H. L. Schreyer and D. L. Sulsky are gratefully acknowledged. This work was supported by the United States Department of Energy through Sandia National Laboratories under Contract DE-AC04-94AL85000. Sandia is a multiprogram laboratory operated by Sandia Corporation, a Lockheed Martin Company, for the United States Department of Energy. 


\section{References}

[1] W. J. Plumbridge, "Solders in electronics," J. Mater. Sci., vol. 31, pp. 2501-2514, 1996.

[2] D. R. Frear, H. Morgan, S. Burchett and J. Lau, The Mechanics of Solder Alloy Interconnects, Van Norstrand Reinhold, New York, 1994.

[3] J. H. Lau, Solder Joint Reliability: Theory and Applications, Van Nostrand Reinhold, New York, 1991.

[4] D. Tribula, D. Grivas, D. R. Frear and J. W. Morris, Jr., "Microstructural observations of thermomechanically deformed solder joints," Welding Res. Supp., pp. 404s-409s, October 1989.

[5] W. M. Wolverton, "The mechanisms and kinetics of solder joint degradation," Brazing and Soldering, vol. 13, pp. 33-38, 1987.

[6] H. J. Frost and M. F. Ashby, Deformation Mechanism Maps, Pergamon, Oxford, 1982.

[7] M. C. Abeyta and Y.-L. Shen, research in progress.

[8] T. Ostrum, "Aging in solder joints from thermomechanical loading," Report of University of Michigan-Dearborn, November 1997, No.5 (Part II).

[9] Z. Guo and H. Conrad, "Effect of microstructure size on deformation kinetics and thermomechanical fatigue of 63Sn37Pb solder joints," J. Electronic Packaging, vol. 118, pp. 49-54, 1996.

[10] B. T. Lampe, "Room temperature aging properties of some solder alloys," Welding Res. Supp., pp. 330s-340s, October 1976.

[11] E. C. Cutiongco, S. Vaynman, M. E. Fine and D. A. Jeannotte, "Isothermal fatigue of $63 \mathrm{Sn}$ 37Pb solder," J. Electronic Packaging, vol. 112, pp. 110-114, 1990.

[12] ASM Handbook, Vol. 2, 10 ${ }^{\text {th }}$ edn., ASM International, Materials Park, Ohio, 1990.

[13] ABAQUS, Version 5.7, Hibbit, Karlson and Sorensen, Inc., Pawtucket, Rhode Island.

[14] G. Bao, J. W. Hutchinson and R. M. McMeeking, "Particle reinforcement of ductile matrices against plastic flow and creep," Acta Metall. Mater., vol. 39, pp. 1871-1882, 1991.

[15] Y.-L. Shen, M. Finot, A. Needleman and S. Suresh, "Effective plastic response of two-phase composites," Acta Metall. Mater., vol. 43, pp.1701-1722, 1995. 


\section{Figure Captions}

Fig. 1 Schematics of the (a) fine and (b) coarse structures used in the modeling.

Fig. 2 Macroscopic stress-strain response during the first cycle of pure shear loading.

Fig. 3 Contours of constant effective plastic strain at the end of the first cycle in pure shear loading, within the (a) $\mathrm{Sn}$-rich phase and (b) $\mathrm{Pb}$-rich phase in the fine structure.

Fig. 4 Contours of constant effective plastic strain at the end of the first cycle in pure shear loading, within the (a) Sn-rich phase and (b) $\mathrm{Pb}$-rich phase in the coarse structure.

Fig. 5 Variations of the local maximum effective plastic strain with the applied macroscopic strain during the first cycle of pure shear loading.

Fig. 6 Macroscopic stress-strain response during the first cycle of uniaxial loading.

Fig. 7 Variations of the local maximum effective plastic strain with the applied macroscopic strain during the first cycle of uniaxial tensile loading.

Fig. 8 Macroscopic stress-strain response in uniaxial tensile loading, with the $\mathrm{Pb}$-rich phase taken to be a purely elastic inclusion phase and an elastic-plastic inclusion phase with yield strength $40 \mathrm{MPa}$. 
(a)

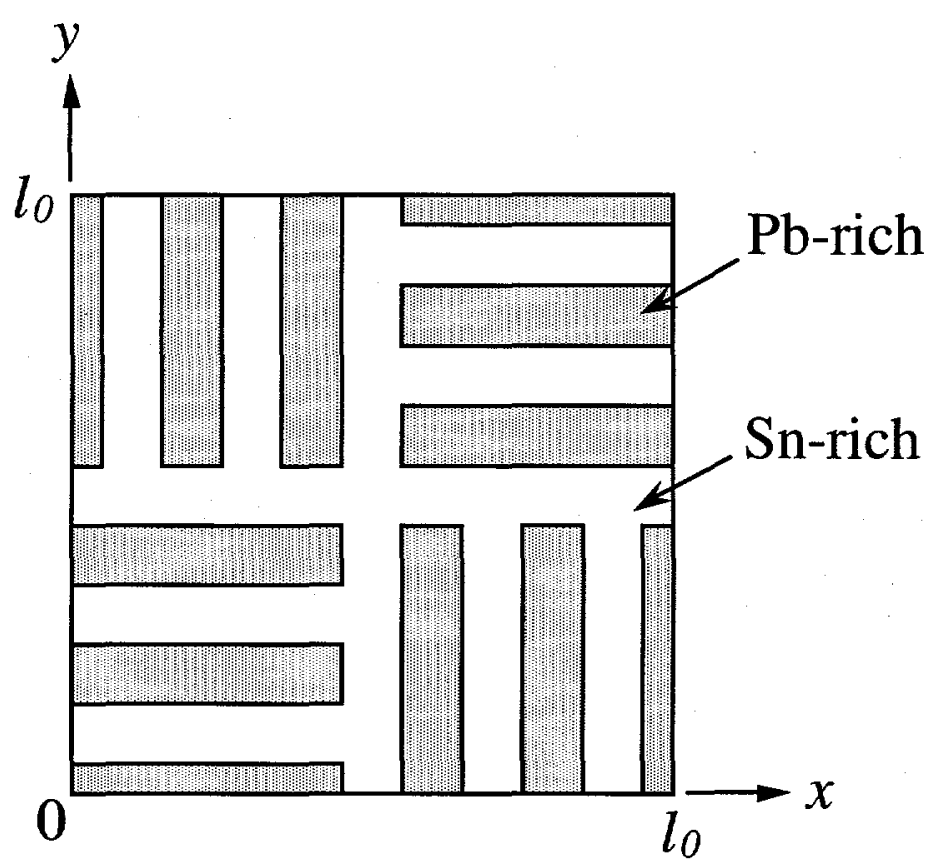

(b)

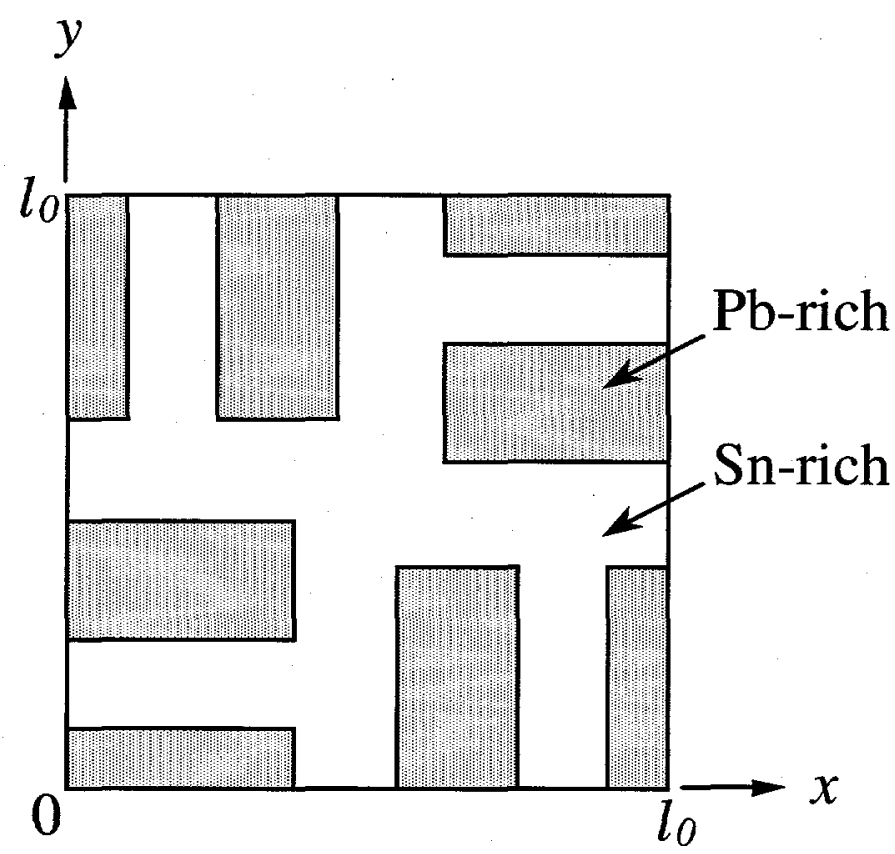

Fig. 1 Schematics of the (a) fine and (b) coarse structures used in the modeling. 


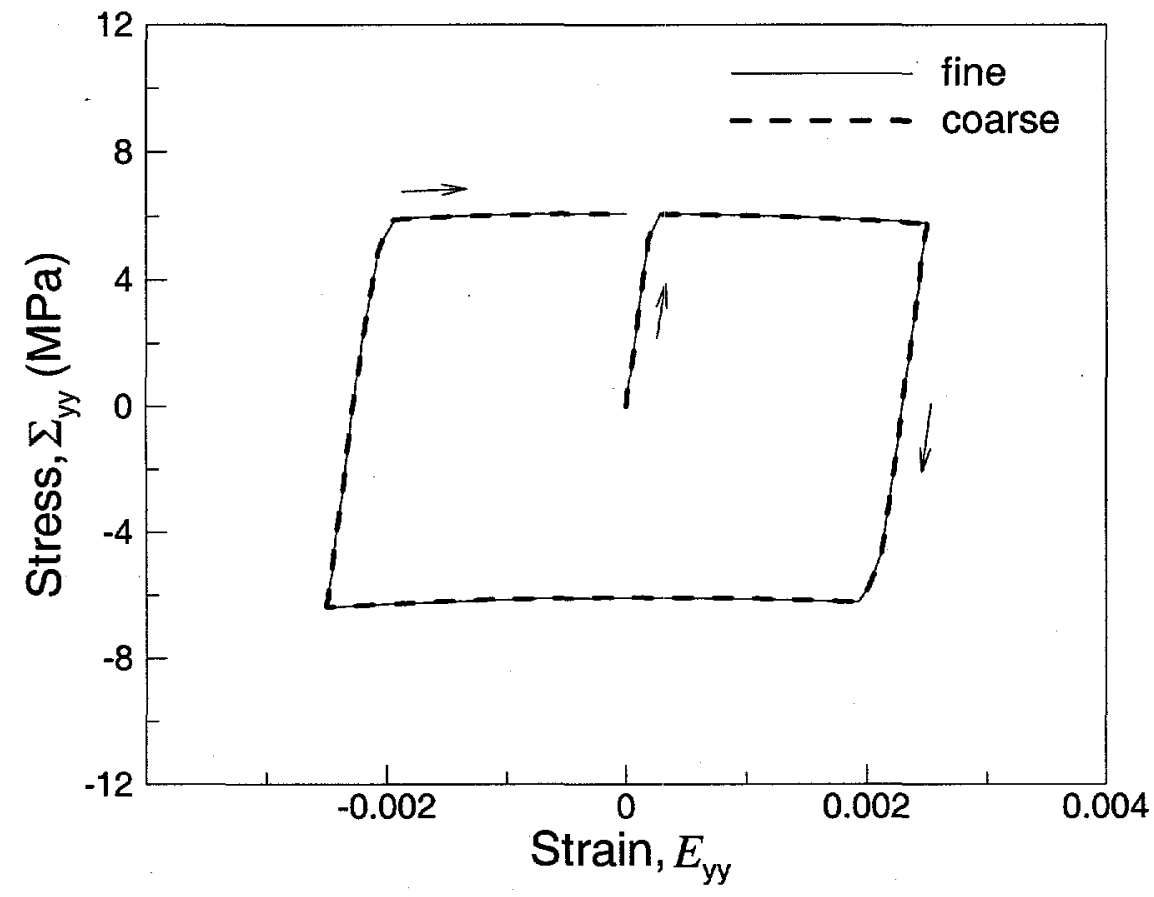

Fig. 2 Macroscopic stress-strain response during the first cycle of pure shear loading. 
(a)
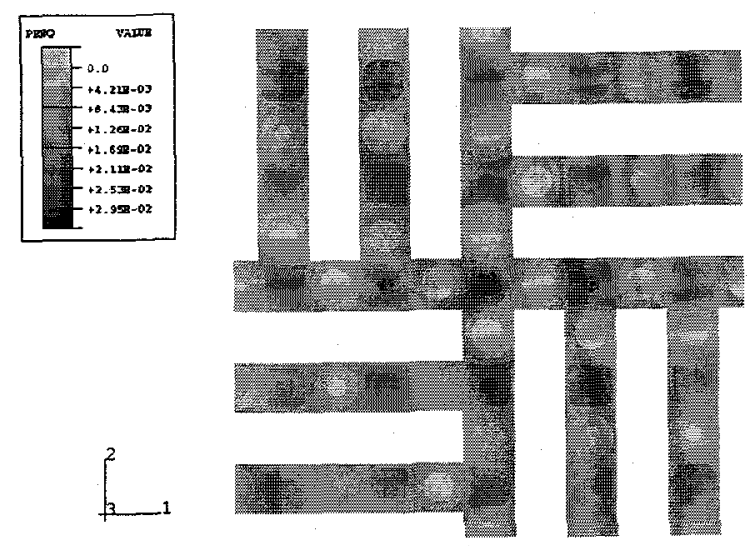

(b)
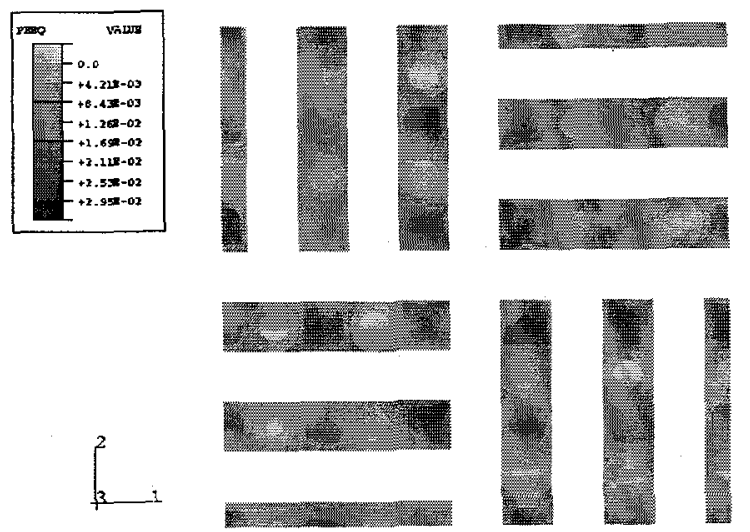

Fig. 3 Contours of constant effective plastic strain at the end of the first cycle in pure shear loading, within the (a) $\mathrm{Sn}$-rich phase and (b) $\mathrm{Pb}$-rich phase in the fine structure. 
(a)
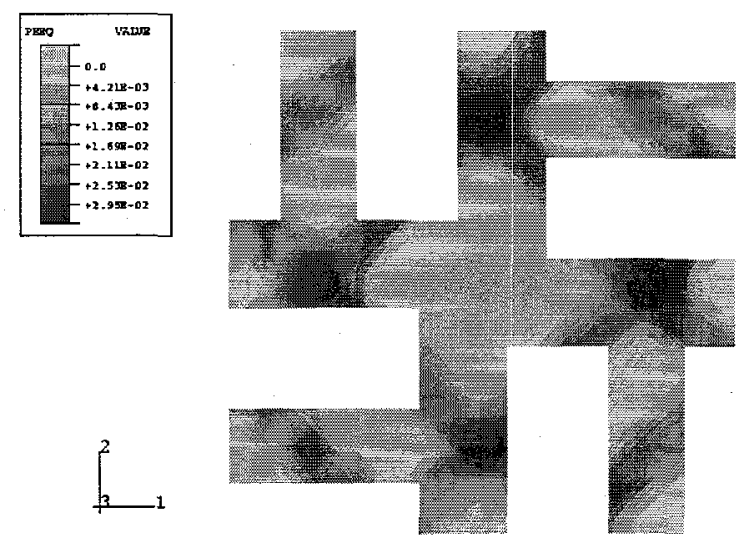

(b)

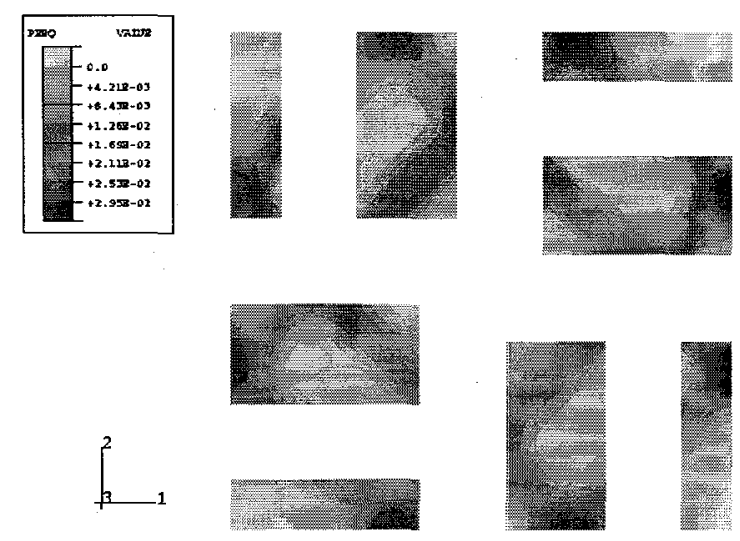

Fig. 4 Contours of constant effective plastic strain at the end of the first cycle in pure shear loading, within the (a) $\mathrm{Sn}$-rich phase and (b) $\mathrm{Pb}$-rich phase in the coarse structure. 


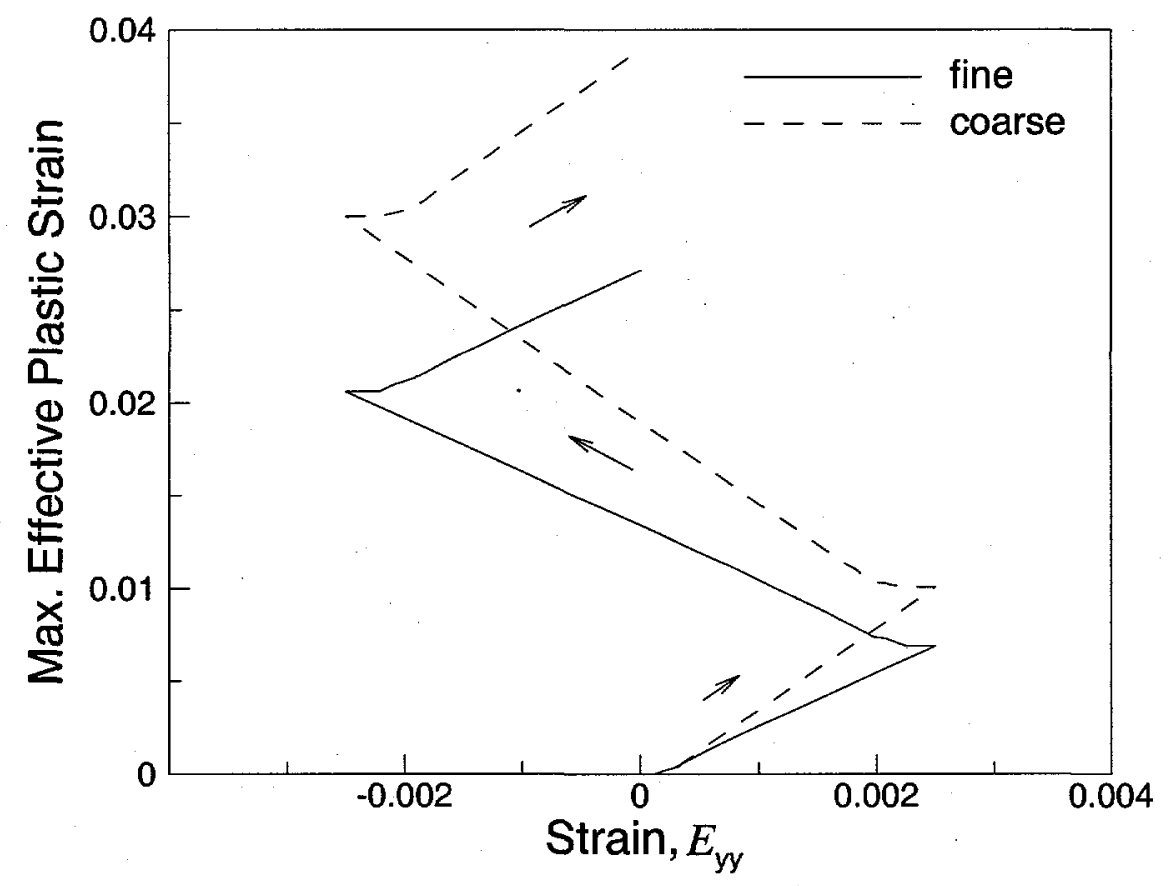

Fig. 5 Variations of the local maximum effective plastic strain with the applied macroscopic strain during the first cycle of pure shear loading. 


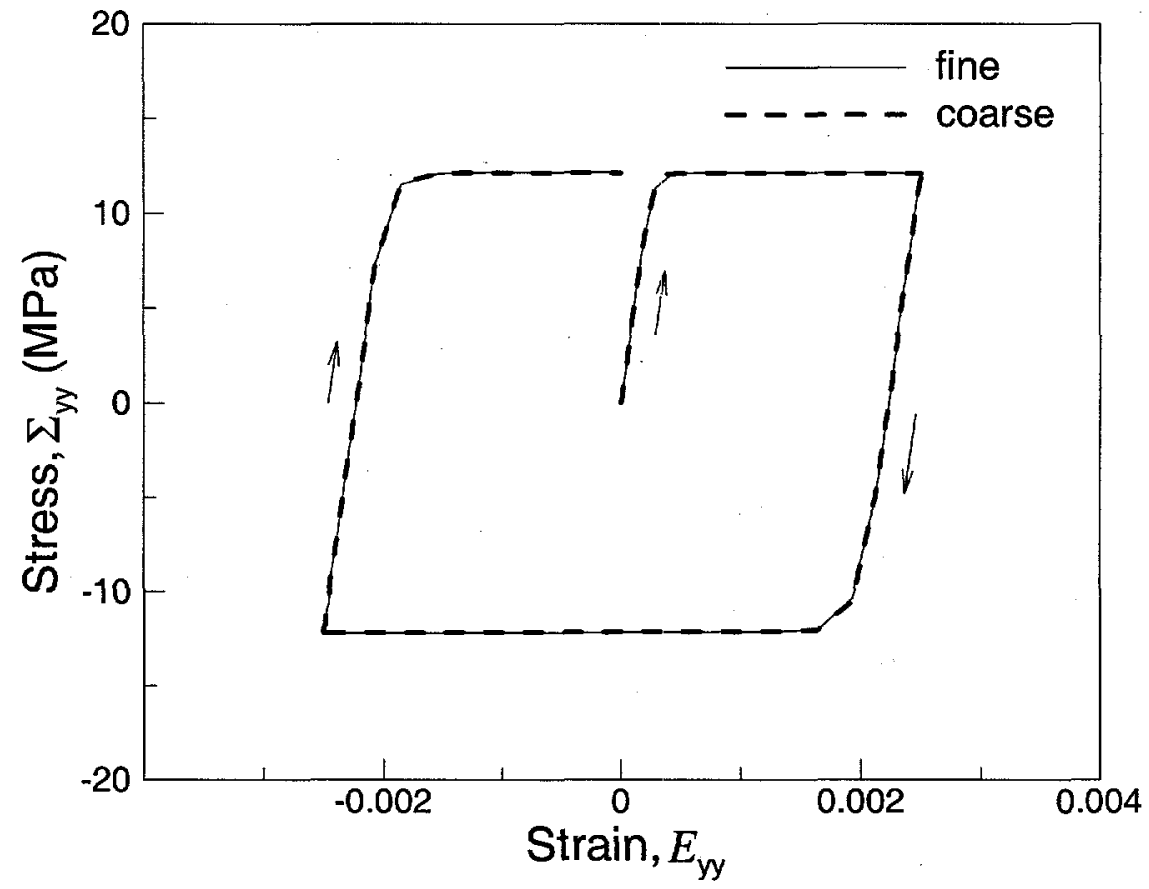

Fig. 6 Macroscopic stress-strain response during the first cycle of uniaxial loading. 


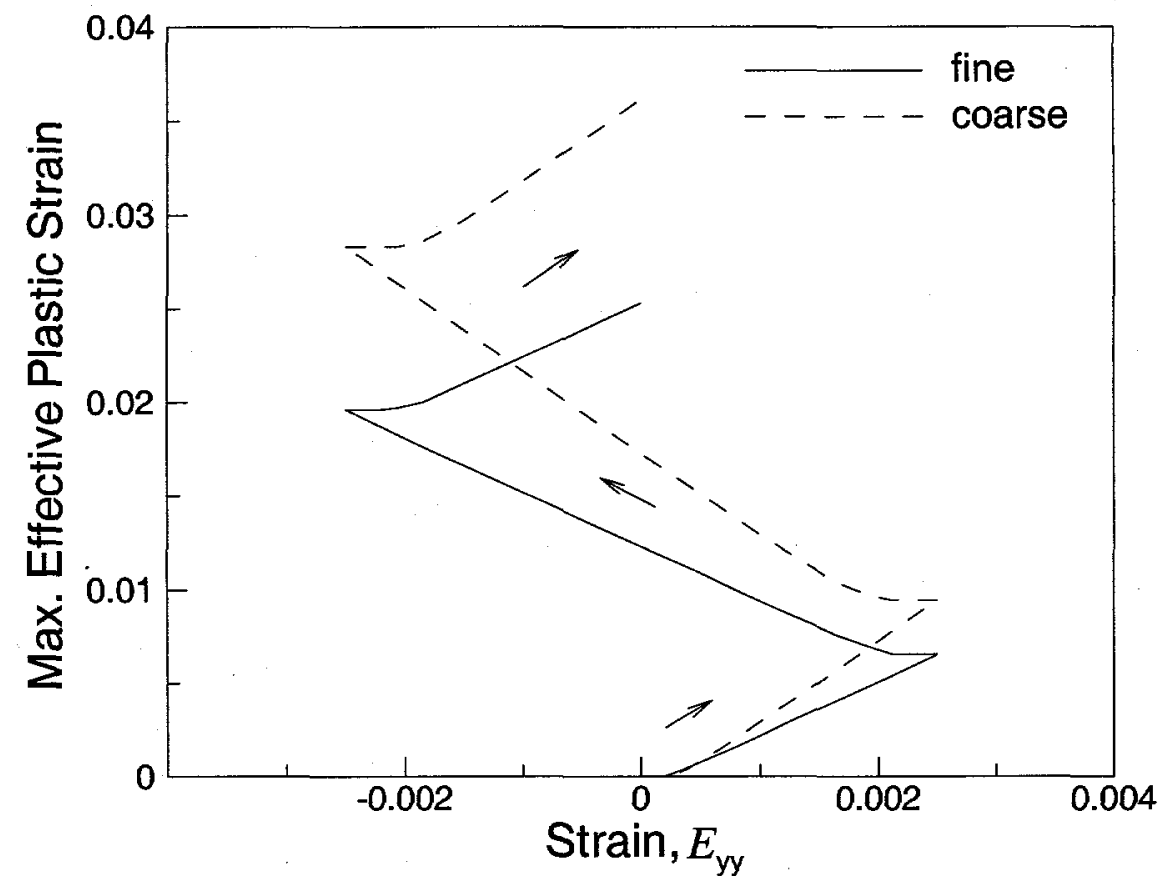

Fig. 7 Variations of the local maximum effective plastic strain with the applied macroscopic strain during the first cycle of uniaxial tensile loading. 


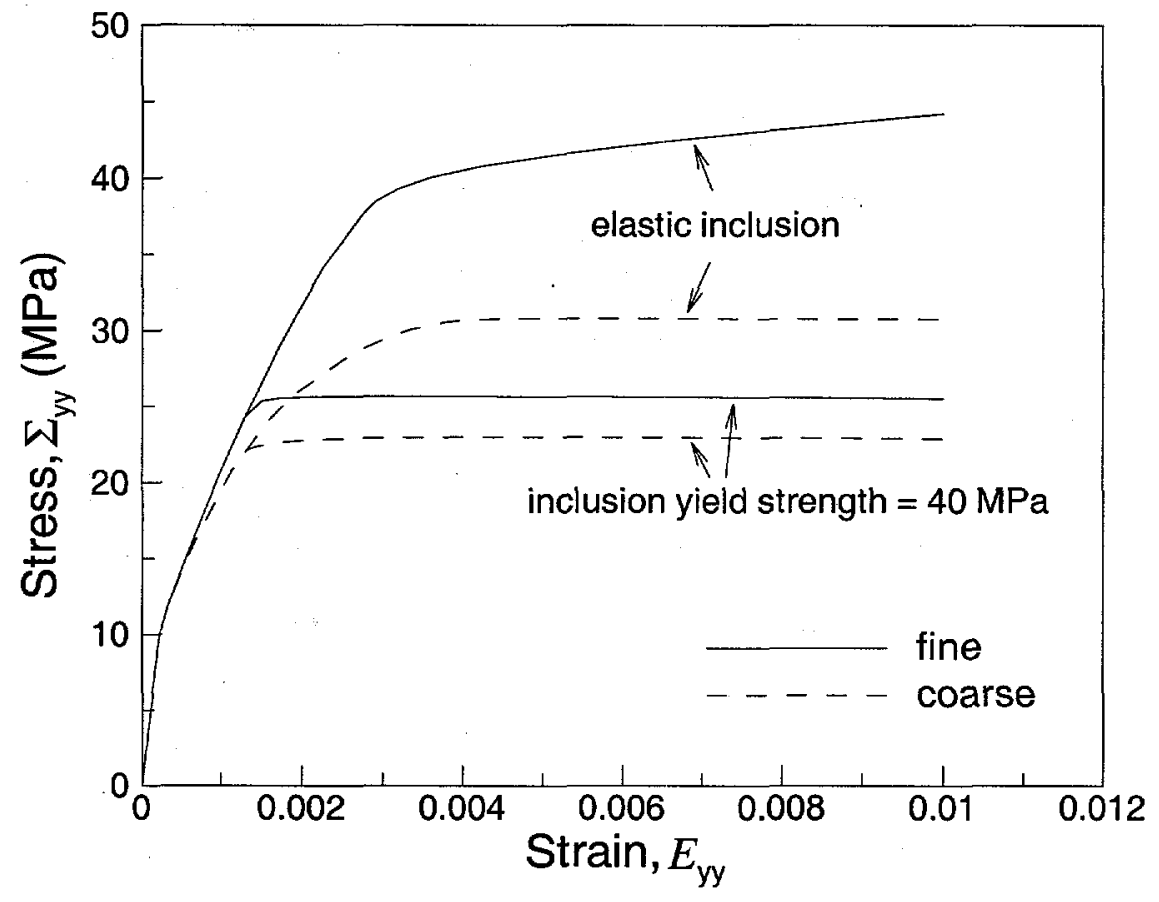

Fig. 8 Macroscopic stress-strain response in uniaxial tensile loading, with the $\mathrm{Pb}$-rich phase taken to be a purely elastic inclusion phase and an elastic-plastic inclusion phase with yield strength $40 \mathrm{MPa}$. 